\title{
History of spectroscopy and modern micromachined disposable Si ATR-IR spectroscopy
}

\author{
Mert Koç and Engin Karabudak \\ Chemistry Deparment, Faculty of Science, Izmir Institute of Technology, Izmir, Turkey
}

\begin{abstract}
In this article, the historical development of spectroscopy is examined and the spectroscopy devices used today are described. Then, we focus on infrared (IR) spectroscopy, which cannot give valuable signal in aqueous solution. Attenuated total reflection (ATR)-IR technique solves the problem. In addition, we specifically mention newly developed disposable ATR-IR crystals and micromachined silicon (Si) ATR-IR. Disposable crystal systems and microfluidics systems can be integrated with existing miniature ATR analyzers. If the integration is successful, the technique might be used in biomedical measuring instruments, reactions' analyses, and ultra-high-pressure analyses.
\end{abstract}

\section{KEYWORDS}

Infrared spectroscopy;

spectroscopy

\section{History of spectroscopy}

\section{Theory of light and matter}

Light has fascinated human kind for centuries. In the history of science, the systematic study of light started with Galileo (1564-1642), who used lenses to observe space. Then, Newton (1643-1727) understood light as a color spectrum. Maxwell (1831-1879), in turn, clarified that light included both magnetic and electric fields. Einstein (1879-1955) conceptualized the idea that light exists as particles. Finally, Feynman (1918-1988) developed electrodynamic diagrams. Figure 1 presents the scientists who contributed to the light theory we know today.

\section{The duality of light as particle and wave}

Through the ages, many experiments tried to discern the nature of light. Galileo and other scientists made futile attempts to measure its speed. Then, Newton explained light's behavior with the idea that light was made of particles. His famous prism experiments divided light into a color spectrum (Figure 2). He passed a beam from a light source through a prism to separate light into six colors. Figure 2 shows Newton's prism experiment $(1,2)$.

On the other hand, Maxwell defined light as an electromagnetic wave. Maxwell's equations (Equation (1)) describe electromagnetism by combining electricity, magnetism, and

CONTACT Engin Karabudak enginkarabudak@iyte.edu.tr $\mathbf{O}$ Chemistry Deparment, Faculty of Science, Izmir Institute of Technology, İzmir 35430, Turkey.

Color versions of one or more of the figures in the article can be found online at www.tandfonline.com/laps.

(c) 2018 Taylor \& Francis Group, LLC 


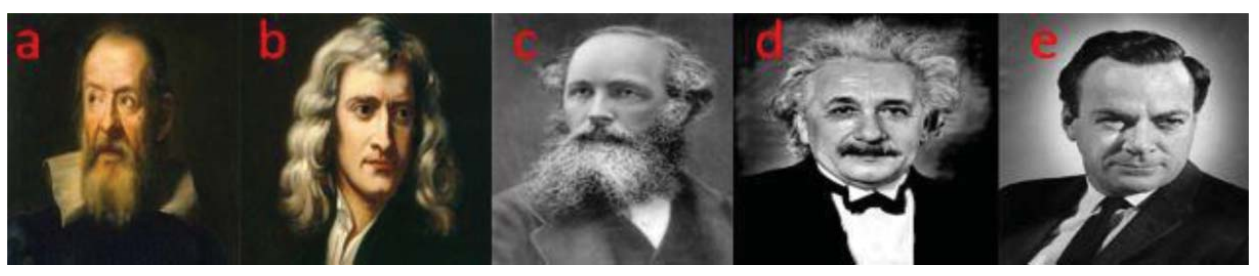

Figure 1. Scientists that developed light and wave theories: (a) Galileo 1564-1642, (b) Newton 16431727, (c) Maxwell 1831-1879, (d) Einstein 1879-1955, and (e) Feynman 1918-1988.

their propagation speed (see Figure 3). Then, Einstein used the photoelectric effect to observe that light consists of energy packages called photons, whose energy depends on the frequency of light (1-3).

However, a paradox emerged concerning why light has wave properties in addition to photon properties. The debates on this paradox resulted in several experiments showing that light has both wave and particle properties (1-3).

$$
\left(c^{2} \nabla^{2}-\frac{\partial^{2}}{\partial t^{2}}\right) E=0\left(c^{2} \nabla^{2}-\frac{\partial^{2}}{\partial t^{2}}\right) B=0
$$

where

$$
c=\frac{1}{\sqrt{\mu_{0} \varepsilon_{0}}}
$$

Equation (1) is the electromagnetic wave equation, where $c$ is the speed of light, $\nabla$ is the Laplace operator, $t$ is the time, $E$ is the magnetic field, and $B$ is the magnetic flux density.

Due to emission and absorption properties, light manifests particle-like behavior. The energy carried by light waves is packaged in discreet bundles called photons or quanta $(2,3)$.

\section{Particle and wave duality of matter?}

From as far back as the ancient Greeks and Romans, philosophers have tried to define matter. According to classical physics, matter is a particle with a definite position and velocity. Atoms are composed of neutrons and protons in the nuclei and electrons in the orbitals. This theory was sufficient for heavy and slow matter with small particle wavelength. However, classical physics could not explain why electrons whose direction continuously changes do not emit electromagnetic waves and do not collapse into the nucleus. Quantum physics explained this phenomenon with the wave function of matter. Electrons remain in their orbitals and do not collapse into the protons thanks to their wave function, described by the

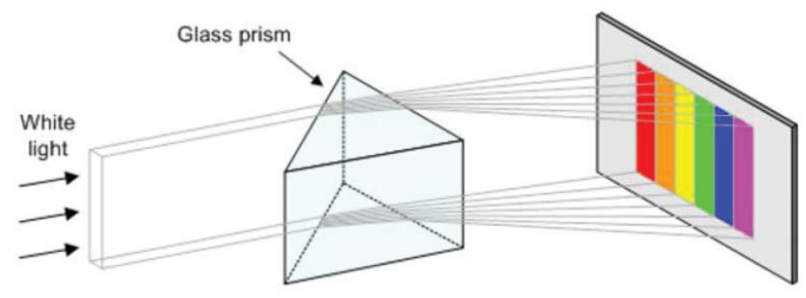

Figure 2. Newton's experiment for splitting white light into a spectrum. 


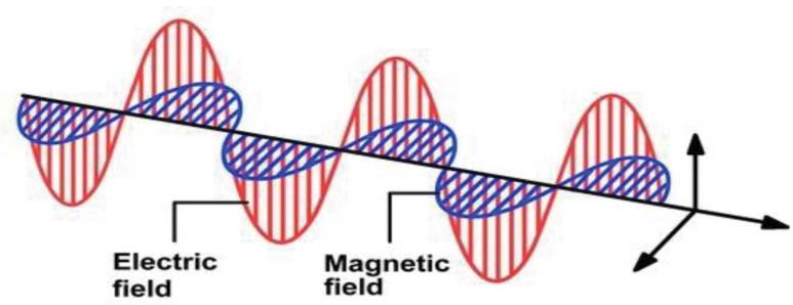

Figure 3. Electromagnetic wave.

Schrödinger equation. This wave function helps describe particles with very small mass and rapid velocity.

According to quantum physics, light, like matter, has both wave and particle properties (Figure 4). The only difference is that matter has mass but light does not. Schrodinger's equation (Equation (2)) describes the electron's wave function, which classical physics could $\operatorname{not}(2,3)$ :

$$
\begin{gathered}
H \psi=E \psi \\
i \hbar \frac{\partial \Psi}{\partial t}=\hat{H} \\
\left(-\frac{\hbar^{2}}{2 m} \nabla^{2}+V\right) \psi=\iota \hbar \frac{\partial \psi}{\partial t}
\end{gathered}
$$

Equation (2) is the Schrodinger equation, where $\Psi$ is the wave function, $E$ is the energy of the state $\Psi, H$ is the Hamiltonian operator, $t$ is the time, $h$ is Planck's constant, $\nabla$ is the Laplace operator, and $m$ is the mass of particle

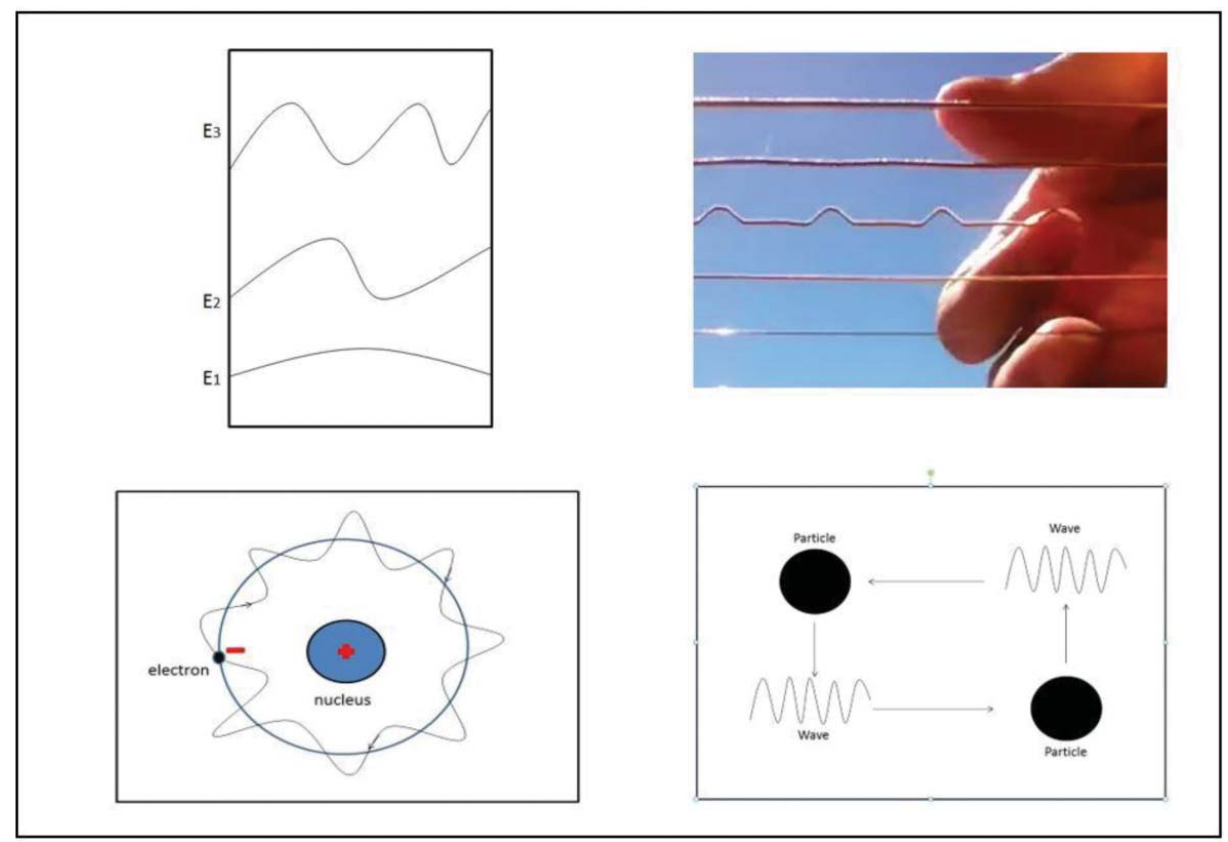

Figure 4. Particle-wave duality. 


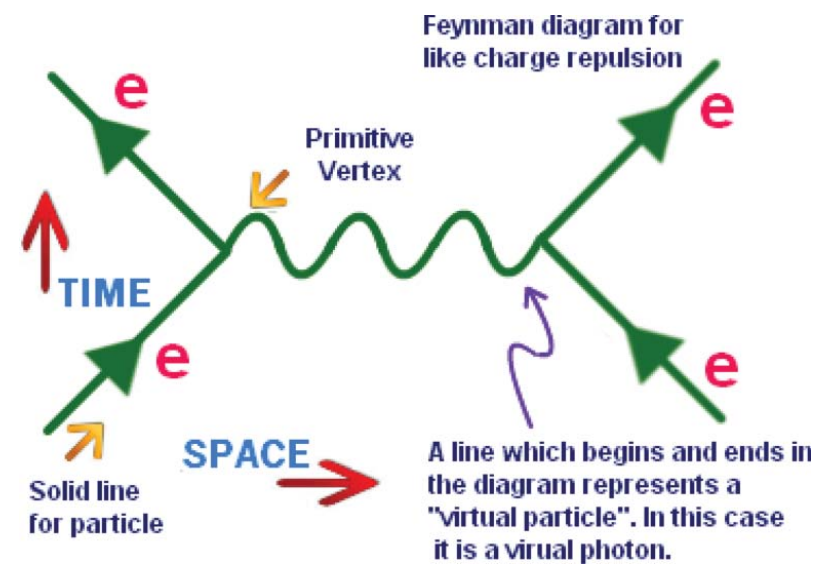

Figure 5. Feynman diagram.

In the early 1930s, physicists tried to develop a new mathematical model to identify radiation and absorption of charged particles. Their theories proved insufficient, providing only discursive or infinite results against strong physical questions. Feynman was attracted by the problem of a stable QED theory in the late 1940s. The problem called for complex mathematical equations. However, Feynman followed a different path from his colleagues. He developed a quantum electrodynamics diagram (Figure 5) that illustrates what happens when subatomic particles interact with each other. Nowadays, it is commonly used as a tool to visualize complex calculations in physics in a simplified way. The diagram in Figure 5 shows that as two electrons move through space and evolve in time, they interact and exchange a photon. The magnitude of the force can be calculated by using Feynman calculus.

\section{Theory of light and matter interactions: Spectroscopy theory}

In short, spectroscopy is the study of interactions between light and matter. When electromagnetic radiation hits and interacts with a sample (Figure 6), absorption, emission,

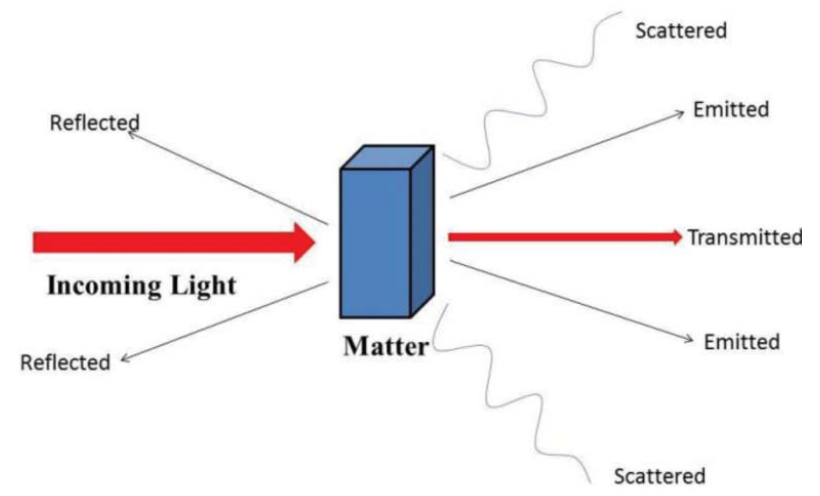

Figure 6. Light and matter interaction. 
reflection, and transmission can be observed $(4,5)$. When radiation interacts with a sample, the electrons interact with light and are excited to a higher energy state from a lower energy state, and absorption is observed. Emission is observed if the excited electrons emit photons while relaxing to a lower energy state. Reflection can occur depending on physical specifications of the sample surface, and medium difference is the most common and effective reason for this deflection. If the sample is transparent in the specified wavelength of the light, it will pass through the sample and transmission will be observed $(4,5)$.

When a photon strikes the outer layer of an atom, it is absorbed by an electron only if the amount of energy it carries is exactly equal to energy differences between high and low quantum energy levels. The energy carried from the light also affects the electrons of an atom in different ways that are explained by the Jablonski diagram. Different spectroscopic techniques analyze in principle different photon and electron interactions in molecules and atoms. So, spectroscopy gives valuable, sensitive, and specific information about atoms, orbitals, bonds, and matter (3-6).

Figure 7 shows that fluorescence occurs if the proton emission exists between states of the same spin state. See for example $S_{1} \rightarrow S_{0}$, where $S_{0}$ is the ground state of a molecule and $S_{n}$ refers to the $n$th excited single state. When the spin states of the first and the last energy levels are different, the emission is called phosphorescence. See for instance $T_{1} \rightarrow S_{0}$, where $T_{n}$ refers to $n$th excited triplet state. As shown in the diagram, this is indicated by a longer wavelength and, therefore, a shorter red line. The lifetimes of fluorescent states are relatively short $\left(1 \times 10^{-5}\right.$ to $\left.10^{-8} \mathrm{~s}\right)$ compared to phosphorescence $\left(1 \times 10^{-4} \mathrm{~s}\right.$ to minutes or even hours), because fluorescence is statistically much more possible than phosphorescence for most molecules. In the diagram, photon emitters are shown as flat arrows, while wavy arrows refer to the non-radioactive process $(1,4-7)$.

Internal conversion (IC), intersystem crossing (ISC), and vibrational relaxation are three non-radiant deactivation processes. The diagram shows examples of these three processes. IC occurs between energy states, which are in the same spin state. ISC occurs between different spin states. Vibrational relaxation occurs between vibrational states in the same energy state and spin. It happens quickly $\left(<1 \times 10^{-12} \mathrm{~s}\right)(1,4-7)$.

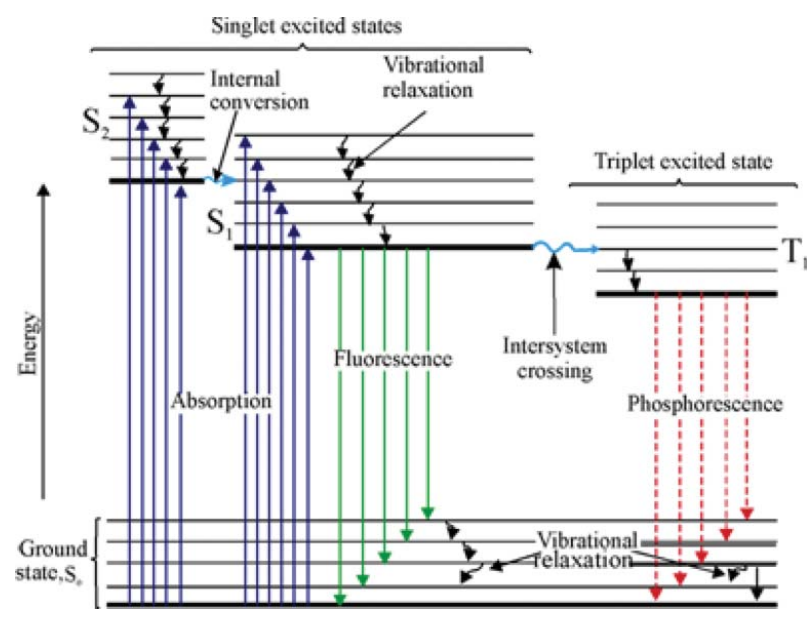

Figure 7. Jablonski diagram. 


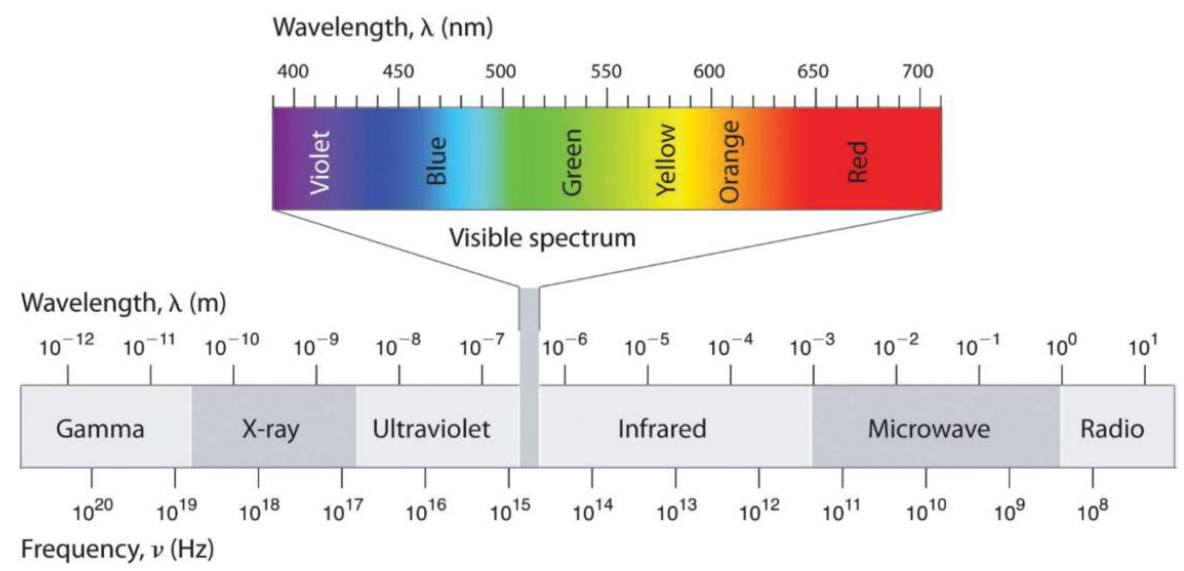

Figure 8. Electromagnetic spectrum (8).

\section{Spectroscopy with different wavelength regions}

The electromagnetic spectrum (Figure 8) comprises all electromagnetic waves of various frequencies and wavelengths that come from light sources. The wavelengths and frequencies of the electromagnetic spectrum range from the nanometer to the meter scale. A very small range of wavelengths can be seen by the human eye, and are called visible light. They can appear in different colors depending on the wavelengths and frequencies of the light $(1,3,4,6)$.

Spectrometers are used to analyze spectrums. A general block body diagram is shown in Figure 9 for spectrometers. In the following sections of this review, we will focus on ultraviolet-visible (UV-Vis), Raman, and infrared (IR) spectroscopies.

\section{Ultraviolet-visible spectroscopy}

UV-Vis spectroscopy occurs in a narrow range of the electromagnetic spectrum, and consists of visible and ultraviolet light. The visible spectroscopy consists mainly of the molecules or matter that absorb visible light wavelengths and frequencies. The specific peaks can be seen by comparing with the standard of the matter and give sufficient information on the characterization of a matter in a mixed solution (4-6).

UV spectroscopy is based on the UV radiation absorption of a sample, occurring from the electron transition from lower electronic states to higher electronic states (Figure 10). There are four types of transitions:

1. $\sigma \rightarrow \sigma^{*}$ transition;

2. $\mathrm{n} \rightarrow \sigma^{*}$ transition;

3. $\mathrm{n} \rightarrow \pi^{*}$ transition;

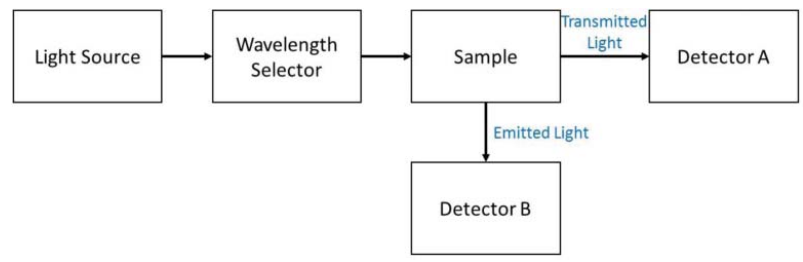

Figure 9. General block body diagram for spectrometer instruments. 
a.

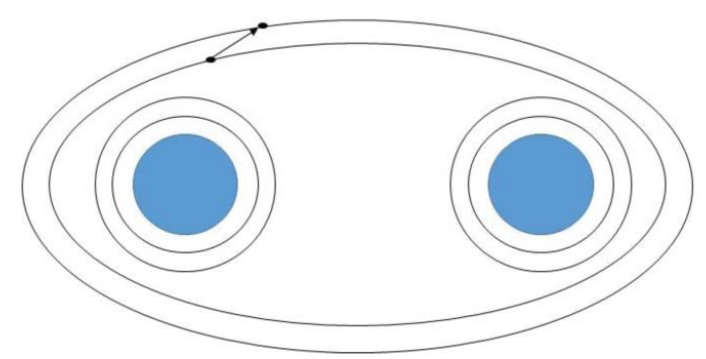

b.

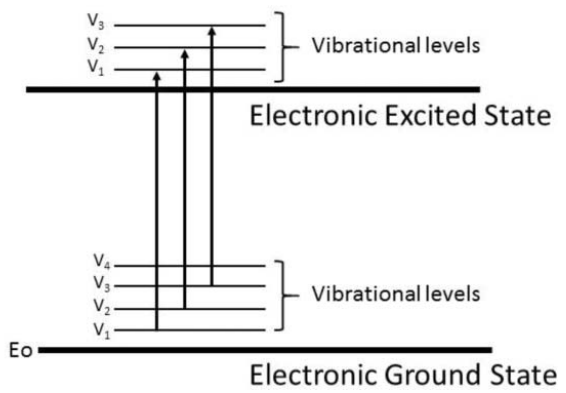

Figure 10. (a) Electron distribution changes on the outer shell. (b) UV spectroscopy transitions on the Jablonski diagram.

4. $\pi \rightarrow \pi^{*}$ transition.

Here, $\sigma$ is a sigma bond, $\sigma^{*}$ is an anti-sigma bond, $\pi$ is a pi bond, and $\pi^{*}$ is an anti-pi bond. These describe molecular orbitals.

UV radiation has large energy, so these transitions can generate wavelengths of 380 $0.6 \mathrm{~nm}$ and from $8 \times 10^{14} \mathrm{~Hz}$ to $3 \times 10^{17} \mathrm{~Hz}$. UV radiation changes electron distribution of atoms or molecules. UV light especially affects microorganisms. The absorbed UV light damages DNA. That is why UV light is used in medicine or related fields for sterilization (4-6).

\section{Raman spectroscopy}

The Indian physicist C.V. Raman discovered that the small amount of light scattered by some molecules differs depending on the wavelength of the incoming beam, and that the wavelength shifts change according to the chemical structure of the scattering molecules (1928). In 1931, he won the Nobel Prize in physics for his discovery and systematic explanations. According to the theory, Raman scattering events are the result of vibration changes with the same type of quantized IR absorption. The wavelength "difference" between the incoming and scattered light falls to the mid-IR region. Raman scattering and IR absorption spectra of a particle are very similar. These two techniques are complementary to each other; an IR active group can be Raman inactive, or a Raman active group can be IR inactive. If the molecule or the vibration mode of a molecule has a dipole moment, then theoretically it can be detected by IR spectroscopy; on the other hand, if the molecule or its vibrational modes has no dipole moment, it can theoretically be detected by Raman spectroscopy $(7,9)$. Figure 11 shows raman transitions and a vibrating molecule.

Some of the advantages of Raman spectroscopy are that it is a non-destructive method, it is used to analyze low wavenumber regions, and it can be used to measure high-temperature-dependent studies. Preparation of the sample is remarkable since even water can be used as a solvent (5). Moreover, Raman spectroscopy can be used with microscopic techniques to achieve better focusing. Small sample volumes can be used. Raman spectroscopy has the capability of light-fiber, which allows a larger separation of the sensing probe and the spectrometer (10). It gives specific information about the molecular structure of biological samples without any labeling, in comparison to standard techniques such as optical microscopy, fluorescence spectroscopy, and immunoassays. The groups of the molecules are especially important for their Raman active or IR active properties (5). Raman spectroscopy helps with bonds and molecules where dipole moment is absent or weak $(5,7,10,11)$. 

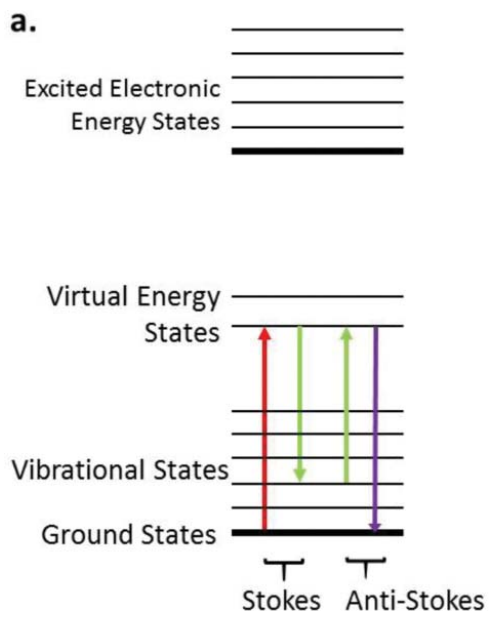

b.

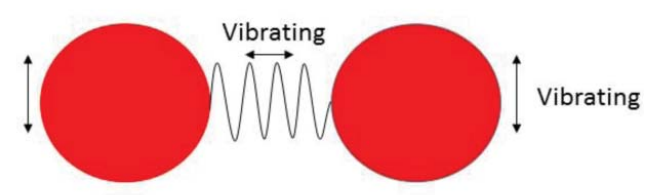

Figure 11. (a) Raman spectroscopy transitions on the Jablonski diagram. (b) Vibrating molecule.

\section{IR spectroscopy}

IR spectroscopy is a well-known and routinely used technique also known as vibrational spectroscopy. Samples must have a molecular dipole moment in order to be studied by IR spectroscopy. Solid, liquid, or gas samples can be analyzed by IR spectroscopy. The molecules in the sample can be identified by determining the chemical structures according to the frequencies of the absorbed IR radiation. The absorbed IR radiation can be detected in the IR spectrum between the area of 12,800 and $200 \mathrm{~cm}^{-1}$, which is divided into far-, mid-, and near-IR spectra. The most important region for identification is the mid-IR region, in which stretching, bending, vibrational, and rotational modes are observed (Figure 12) (4-6).

Vibrational or rotational modes can be calculated theoretically using the formula:

$$
\begin{aligned}
& \text { Linear Molecules Modes }=3 \times n-5 \\
& \text { Nonlinear Molecules Modes }=3 \times n-6
\end{aligned}
$$

where $n$ is the number of atoms in the molecule. Not all of these calculated modes can be seen in the IR spectrum because they must be IR active. IR active means the molecule should have dipole moment change.

a.

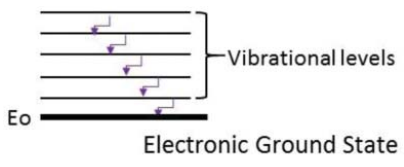

b.

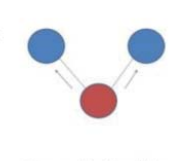

Symmetric Streching

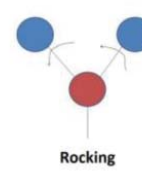

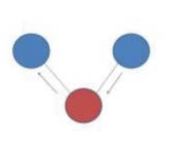

Asymmetric Streching

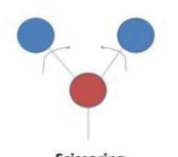

Scissoring
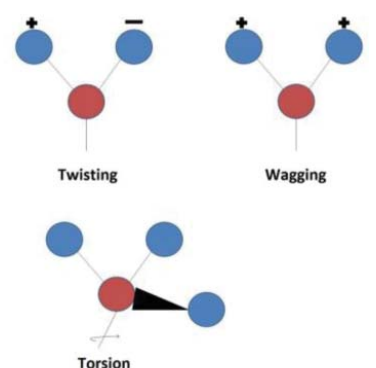

Torsion

Figure 12. (a) IR spectroscopy transitions on the Jablonski diagram. (b) Vibrational modes. 


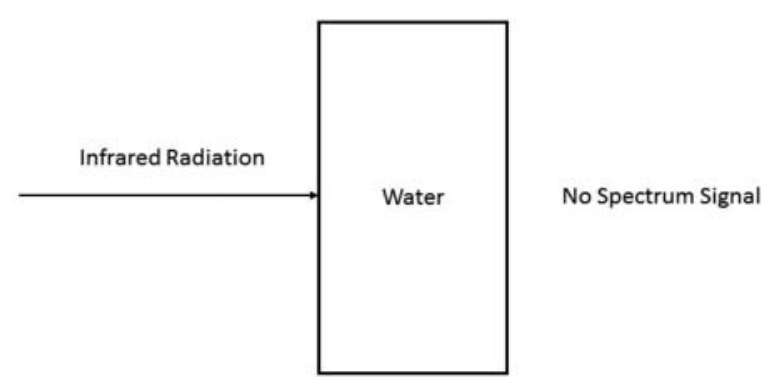

Figure 13. Main disadvantage of IR transmission spectroscopy. Strong IR absorption of water prevents use of IR transmission mode in aqueous solutions.

\section{Limitations of IR and Raman spectroscopy}

IR and Raman spectroscopy are widely used in the fields of drug development and drug testing in order to obtain knowledge of the molecular structure of a sample. The two methods give complementary knowledge about the molecule investigated. The biggest limitation of IR transmission spectroscopy is that strong IR absorption of water molecules prevents accurate usage of IR spectroscopy in aqueous solutions (Figure 13). Thus, in order to analyze aqueous solutions, attenuated total reflection (ATR) technique is used with IR spectroscopy $(4-6,12)$.

\section{ATR-IR spectroscopy}

ATR-IR spectroscopy is a surface characterization technique that was developed nearly half a century ago (13). The IR enters a stationary processed specific ATR crystal, which provides attenuated total reflectance of the IR beam inside the crystal. ATR-IR analysis works well with aqueous samples, and is, therefore, used in many fields of research (4-6).

\section{Principle of ATR-IR}

ATR is used alongside IR spectroscopy to provide reflections of IR beams by using a highly refractive crystal that has an angle greater than the critical angle. This produces internal reflections at the surface of the crystal which is in contact with the sample. These internal reflections (Figure 14) form multiple evanescent waves that penetrate the sample. These evanescent waves enter the sample at a depth of 0.5-2 $\mu \mathrm{m}$, which causes IR radiation and sample interaction, allowing us to record the ATR-IR spectrum of the sample (12).

Mid-IR beams are usually used for ATR-IR applications because of a special region called a fingerprint region. In comparison with other IR wavenumber regions, fingerprint regions are special because they contain molecule-specific molecular vibrations, so each peak corresponds to only one specific molecule $(4-6,12)$.

The critical angle, total reflectance, internal reflection element (IRE), evanescent wave, and penetration depth are important terms in ATR-IR spectroscopy. The most important component of ATR-IR is a polished IRE on which the sample is placed. The light, which passes through the polished IRE, must be reflected and form evanescent waves to interact with the sample at the interface. This is ensured by the high refractive index material of the polished IRE and an incidence angle larger than the critical angle. The attenuation occurs during the interaction of evanescent waves with the sample, and the reflected light is collected by a detector $(5,13-15)$. 


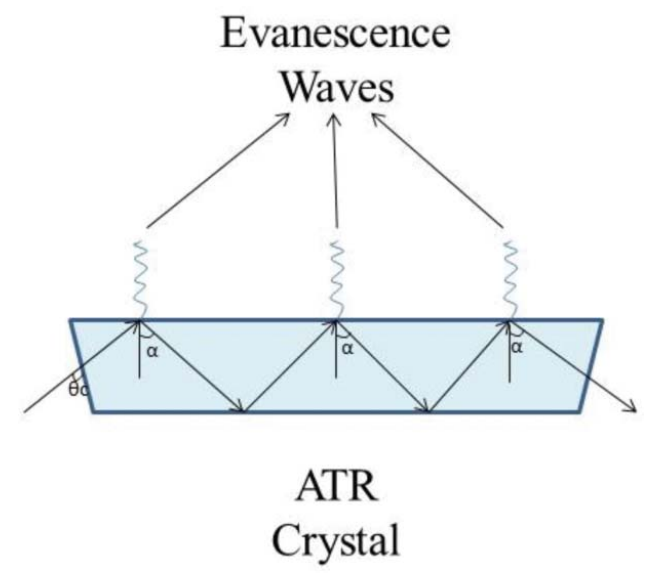

Figure 14. ATR crystal and evanescent waves.

The materials commonly used for ATR crystals are Ge, ZnSe, or diamond. In general, Ge or $\mathrm{ZnSe}$ are used as the raw materials, but they are expensive and usually fixed on the setup in IR spectroscopy. In addition, analyses that require high temperature or highly acidic and basic solutions are harmful to these crystals $(5,13-15)$. Among the three common crystal materials, diamond is the least practical because of its high cost and limited surface area, despite being stable and the most robust. ZnSe is the most affordable material, but can only be used in the $\mathrm{pH}$ range of 5-9 $(5,12-15)$. Today, an emerging alternative IRE is disposable silicon ( $\mathrm{Si}$ ) IRE, which has various advantages that we will discuss in later sections $(5,13-15)$.

\section{Advantages of ATR-IR}

There are three main advantages of the ATR-IR technique in comparison to IR transmission spectroscopy. First, samples can be measured at aqueous solutions, which are not suitable for IR transmission spectroscopy, since large peaks of water prevent accurate measurement in IR transmission spectroscopy $(5,9,10,12-16)$. Second, it is easier to prepare a sample for both liquid and solid materials when compared to IR transmission spectroscopy (16). Third, ATR-IR is suitable to analyze samples' surfaces if it is required for the samples and we can change the penetration depth of evanescent wave by changing the reflection angle (9).

\section{Application of ATR-IR}

ATR-IR is the one of the most important techniques for surface analysis, in which surface absorption, surface thickness, and surface interactions between chemicals and molecules can be determined. In particular, the fingerprint regions give more specific and reliable information about molecules in ATR-IR. As a result, drug-protein interactions and redox reactions can be investigated. Coating thickness and smoothness can be determined (9, 10, 17-29).

One of the most important applications of ATR-IR is the measurement of body fluids, which give possibility of easier sample preparation, working with low volume and nondestructive measurement on sample. At crime scenes, samples of body fluids such as blood, saliva, semen, or vaginal fluid are collected for forensic analyses (30). However, samples may be too limited to analyze repetitively. Developed techniques such as proteomics or mRNA analyses are not yet suitable for general use. ATR-IR spectroscopy also has the capability to 
determine the dissimilarity between biologic molecules such as proteins and their phosphates, sugars by using the finger print region. Moreover, small amounts of sampling are enough for repeat analyses $(26,27,29-31)$.

\section{Disadvantages of ATR-IR}

Sensitivity problem of ATR-IR. ATR-IR works by using evanescent waves, so its sensitivity is not as good as that of the transmission technique. In the transmission technique, light directly passes through the sample, and the detector gathers what comes after sample-light interaction. On the other hand, in the evanescent wave technique, light is projected onto the sample surface and evanescent waves occur in the interface between sample and IRE; hence, light does not pass through the sample, and thus the transmission technique is more sensitive than the evanescent wave technique.

Surface sensitivity. The evanescent wave technique only analyzes a few microns of the sample's surface. The transmission technique allows us to analyze the whole sample (both bulk and surface), but the range of evanescent waves is limitedly adjustable. Some situations require depth to get information about a sample. The ATR is a surface limited technique, so if the sample is not homogeneous in the size of the penetration depth of evanescence wave, we can only get signal from the surface. So, ATR-IR spectroscopy might give different signals compared to the IR transmission technique. This must be taken into consideration when experiments are carried out.

Availability of ATR-IR crystal. Crystals of ATR-IR are not easy to produce and relatively expensive, although they can be used repeatedly. Advanced techniques are needed to produce commercially available crystals, and these processes are costly. Also, they tend to be fragile. In addition, during ATR-IR analysis, the crystal may be damaged if care is not taken. Because the ATR-IR is a surface-sensitive technique, the damaged crystal usually reduces sensitivity.

As explained before, it is necessary to carefully clean the crystal before every single measurement. So, it is not practical to use new crystal for each sample measurement. This may cause sample to sample contamination. In some fields like biomedical applications, cleaning the crystal again and again is not possible, because of the requirement of hygiene. Also, high-temperature processes can damage the crystal, if high-temperature measurement is required in any field.

\section{Modern micromachined disposable Si ATR-IR}

\section{Micromachined ATR-IR}

The science of altering and examining fluids in micron-sized channels or micron-sized chamber is called microfluidics. It is usually practiced in the range of microliters $\left(10^{-6}\right)$ and picoliters, and includes tens to hundreds of micrometer-sized channel networks. Microfluidics was applied to research chemical weapons at the Defense Advanced Research Projects Agency (DARPA) of the US Department of Defense starting in the 70s (32). With the progress of molecular biology in the 80 s, scientists decided to investigate a novel analysis method, since determination of genomes and proteins involved extended steps. In the beginning, a 
single micro-device was generated for the microfluidic chip. However, the major expectation was to perform multiple analyses with the single tool in microfluidics. Also, the analysis was of lower cost. Additionally, when the microfluid chip connected with nanochannels, it allowed the transportation of liquid and the other devices. For instance, gas chromatography, high-performance liquid chromatography, and capillary electrophoresis can be synchronized with the microfluidic chip so that separation can occur in the nanochannels of the microfluidic chip (32).

In addition, microfluidics has become more popular in chemical synthesis. The major advantages of microfluidics include extended experiment time, being faster, and using small quantities of solvents. Using microfluidics is environment friendly due to the small amount of toxic compounds and waste produced. The management of heat of the reaction and mixing product has an important role in synthesis chemistry and microfluid technology supports to keep these parameters under control. Also, formation of by-products is lower than that in macro-scale experiments. This has advantages in quantum dot synthesis. Since the range of quantum dots is desirable in chemistry, the efficiency of reactions is improved. So, the combination of microfluidics technique with the synthesis of quantum dots appears promising (32).

Microfluidics is an important field that can be used in various other applications in order to minimize the experimental space, which is also known as lab-on-chip. Microfluidics has various advantages on various applications, but on the other hand, it is very difficult to detect chemicals in-situ inside the microfluidics channels. Because microfluidics channels have closed geometry, so many of the analytical techniques cannot analyze the chemicals online inside the microchannels. This is why micromachined Si ATR-IR is developed as a novel analytical tool for analyzing the liquids inside micro/nanofluidics in-situ $(13,15)$.

\section{Polished IRE-coupled microfluidics}

Since light travels at different speeds inside different materials, the refractive index of the material is especially important in the field of ATR-IR. Due to the high refractive index of ATR-IR IRE, incoming IR light creates total reflectance inside the IRE material. Therefore, there is no intensity loss during these reflections. For this reason, the type of IRE material used in ATR-IR setups is very important (15).

As mentioned before, IRE material must have a high refractive index and transparency in order to accurately measure wavelengths. In addition, it must be a mechanically processed material to allow formation of a specific shape to determine the appropriate angle of incidence. This is especially important in order to arrange the penetration depth of the evanescent wave. Commercial IREs called polished IREs are produced by mechanical polishing to achieve this specific shape (15). The polished IRE must have a very smooth surface on its reflection side.

The major drawbacks of commercial IREs are their production costs. As an alternative to commercial IREs, $\mu$ Si-IREs are produced as well-developed microfabrication products (13). In fact, $\mu$ Si-IREs can work in the fingerprint region, while commercial IREs cannot. In addition, the disadvantage of Si-IRE (that the working spectral range is limited to a cut-off value below $1,500 \mathrm{~cm}^{-1}$ ) is removed by producing $\mu$ Si-IRE (15).

\section{uSi-ATR-IR-coupled micro/nanofluidics}

The first Si-ATR-IR microfluidics system in the literature was presented in 2004 (33). Anisotropic $\mathrm{KOH}$-etching of a $\mathrm{Si}$ wafer is a certain method to generate optically smooth surfaces 


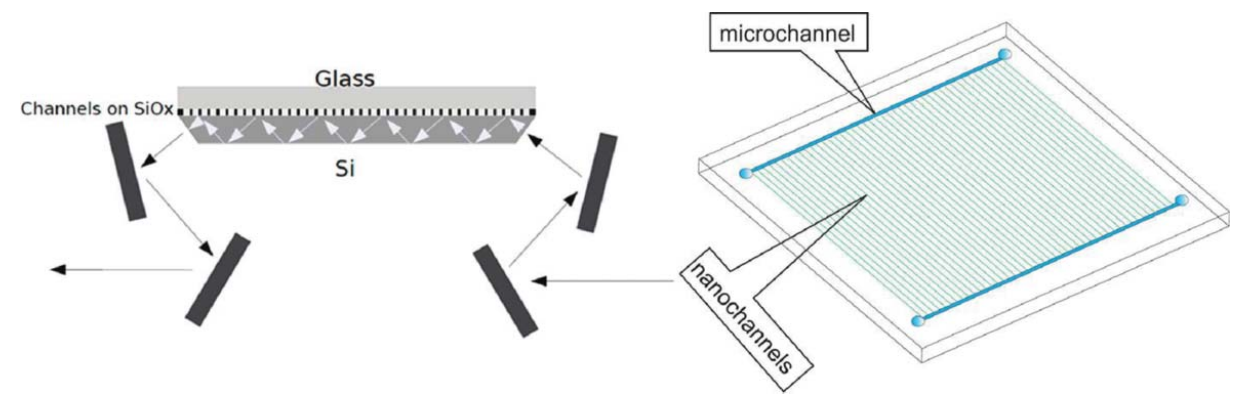

Figure 15. Micromachined Si-ATR IR systems (From Karabudak, Micromachined silicon attenuated total reflectance infrared spectroscopy: An emerging detection method in micro/nanofluidics, Electrophoresis. Copyright 2013 WILEY-VCH Verlag GmbH \& Co. KGaA, Weinheim).

with a Si wafer. It was performed instead of polishing wafers. This demonstrated that $\mu \mathrm{Si}$ IREs can instantly occur from a $100 \mathrm{~mm} \mathrm{Si}$ wafer. Anodic bonding was used to bond the wafer with micromachined channels on top of these Si-IREs. The top surface and connections occurred with PDMS material, as shown in Figure $15(13,15)$.

The whole system was put into a commercial FTIR spectrometer without fabrication of any special optical setup. Fabricated IREs (Figure 16) were produced with a length of $1 \mathrm{~cm}$ so that the probe could measure the fingerprint region. This allowed the examination of a wavenumber as low as $800 \mathrm{~cm}^{-1}$. The study proved for the first time that Si-ATR-IR could examine the chemical reactions in the channels of microfluidic chips $(13,15)$.

\section{Disposable ATR-IR crystals and their importance in chemical analysis}

It is a well-known fact that IR spectrometers are expensive and massive instruments, although technology is improving day by day. One of the reasons is that ATR-IR crystals are neither easily produced nor disposable. The crystals are made of $\mathrm{Ge}, \mathrm{ZnSe}$, or diamond. Generally, if a crystal is placed into the spectrometer system, it will not be replaced until it is broken or contaminated. Because of their price, crystals must be used carefully.

However, a new promising development allows the production of easily replaceable crystals made from a Si wafer. Si wafers are relatively cheap materials compared with Ge, ZnSe, and diamond. Karabudak et al. (13) successfully fabricated disposable Si IREs. In disposable IRE fabrication, a dicer is used in place of the time-consuming $\mathrm{KOH}$-etching process. This allows a huge number of disposable Si IREs to be produced quickly and cost effectively. Therefore, one-time use Si IREs (disposable Si IREs) can be produced. These disposable Si

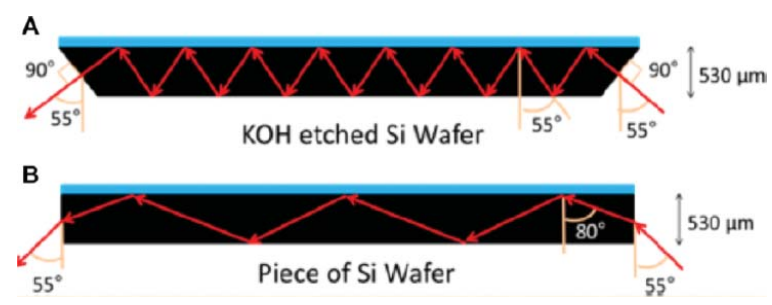

Figure 16. Refractivity of (a) KOH-etched Si wafer. (b) Piece of Si wafer (From Karabudak, Micromachined silicon attenuated total reflectance infrared spectroscopy: An emerging detection method in micro/nanofluidics, Electrophoresis. Copyright 2013 WILEY-VCH Verlag GmbH \& Co. KGaA, Weinheim). 
IREs can have a significant effect on different fields in which ATR-IR is used. They eliminate the need to clean ATR-IR crystals every time, so the sample can be kept with the crystal as long as needed (13). These crystals will be an advantage in the field of biomedical research, where high hygienic conditions are required. Also, highly explosive, highly acidic, and highly basic experiments can be performed. Another important feature is ease of use. When the amount of sample to be examined is small, it can easily be stored on the crystal for reuse. Then, this crystal can be analyzed without any of the problems of other devices, such as scanning electron microscopes (SEMs). It even stood up to a temperature test in the oven up to $1,414^{\circ} \mathrm{C}$.

\section{Miniaturizing of ATR-IR spectrometers}

Many instrumentation companies are trying to make smaller IR spectrometers. Smaller versions of IR spectrometers are announced every year. A review of handheld IR spectrometers was published by Sorak et al. (34). Companies such as Pyroes and Hamamatsu are producing handheld spectrometers. However, the poor spectral quality and high cost of these instruments prohibit their use. Nevertheless, it is most probable that these systems will become more common in analytical labs in the near future if technological developments allow the manufacturing of smaller IR spectrometers with better quality and low cost. Additionally, there have been academic trials to unite whole ATR-IR systems into a single embedded chip, as shown in Figure $15(14,15)$. In these systems, the whole light source and detector are accrued into the same Si wafer. However, they have two major disadvantages. First, these systems do not work as efficiently as commercial IR spectrometers, since the embedded light source and detector are not as efficient as commercial ones. For instance, the embedded light source is not as accurate or intense as commercial light sources. This minimizes the quality of the spectra that are observed. In addition, embedded detectors cannot detect all wavelengths at once. Some specific wave ranges are chosen and the whole system works in these wavelengths. Another drawback is that the manufacturing process of these devices is time consuming, expensive, and complex. The manufacturing process requires clean room facilities, which adds to the cost of the device. Device cost is not a scientific issue; however, it is important to general use of the analytical technique. In brief, technologically, it is possible to integrate a light source and detector into a single Si wafer. However, with current technology, the system efficiency is too low and the cost is too high. If the cost of microfabrication is reduced and the quality of an embedded light source/detector is improved, these kinds of systems can have an important role in the field $(14,15)$.

The company Pyroes started to fabricate handheld mid-IR spectrometers. Portable Pyreos ATR is a small size ATR spectrometer with the dimensions $165 \times 74 \times 35 \mathrm{~mm}^{3}$. ZnSe crystal has been used as an ATR crystal with a surface area of $17 \times 27 \mathrm{~mm}^{2}$. The ATR can scan a range of 1,818-909 $\mathrm{cm}^{-1}$. The company has also been developing kits, one of which was purchased by our laboratory (Figure 17). These systems can analyze samples using mid-IR radiation including fingerprint region. The sample can be easily placed on an ATR-IR crystal, and spectrum can be taken in a second. In addition, it is a small, lightweight, battery powered, low power consumption device that can analyze liquids, solids, and slurries. Figure 17 shows the basic working process of Pyroes ATR and a Pyroes ATR model. The ATR can be easily cleaned, which allows fast and reproducible analysis. Pyreos ATR is used with SphinxSuite Software combined with powerful and easy to use chemometric analysis software (35). 

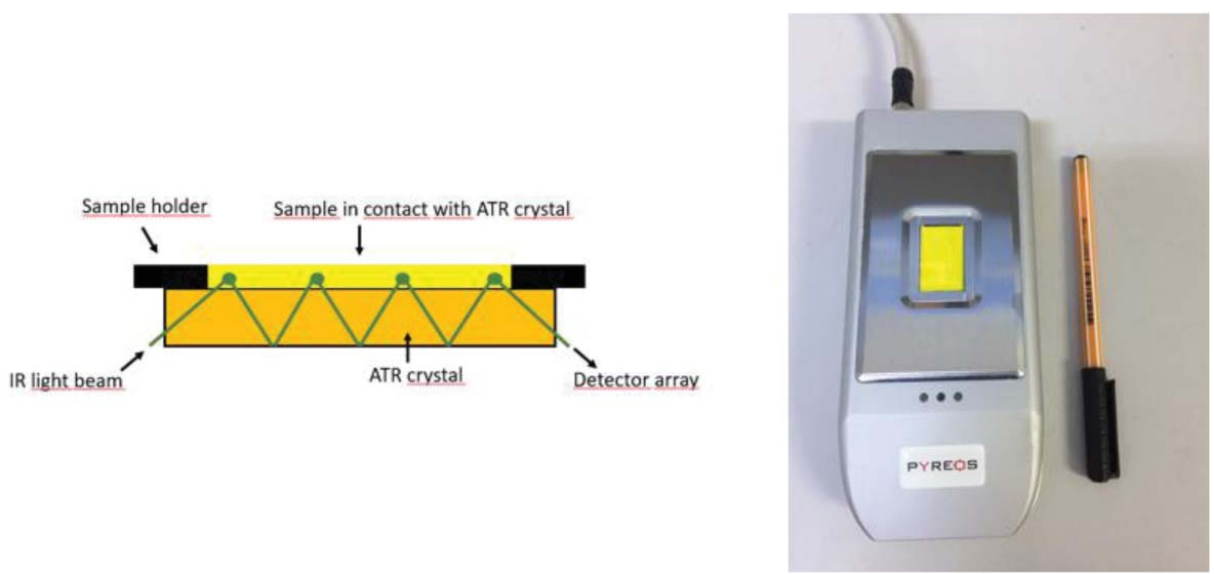

Figure 17. Basic scheme of Pyroes ATR and Pyroes PY0715-ATR (35).

Meanwhile, micro-spectrometers are being improved by other companies. Hamamatsu has developed a finger-tip size, ultra-compact spectrometer head. The device is integrated with micro-electromechanical systems (MEMS) and image sensor technologies. It can be used with printers and a handheld color monitoring device. It can also be combined with mobile devices if a suitable application is used. The device has a $340-780 \mathrm{~nm}$ spectral response range with $15 \mathrm{~nm}$ spectral resolution. Its weight is only $5 \mathrm{~g}$, and its dimensions are $20.1 \times 12.5 \times 10.1 \mathrm{~mm}^{3}$ (Figure 18) (36).

\section{Future perspectives and emerging applications of ATR-IR technology}

Micro-machined ATR-IR is suitable for research on high-pressure catalytic reactions. A high-pressure micro-machined ATR-IR can be developed in the future. Reaction mechanisms at 600 bar might be analyzed. Such technology would be valuable to the petroleum refining processes, for example. An Illustrative drawing is shown in Figure 19. Ultra-high-pressure micromachined Si ATR-IR may play an important role in such research.
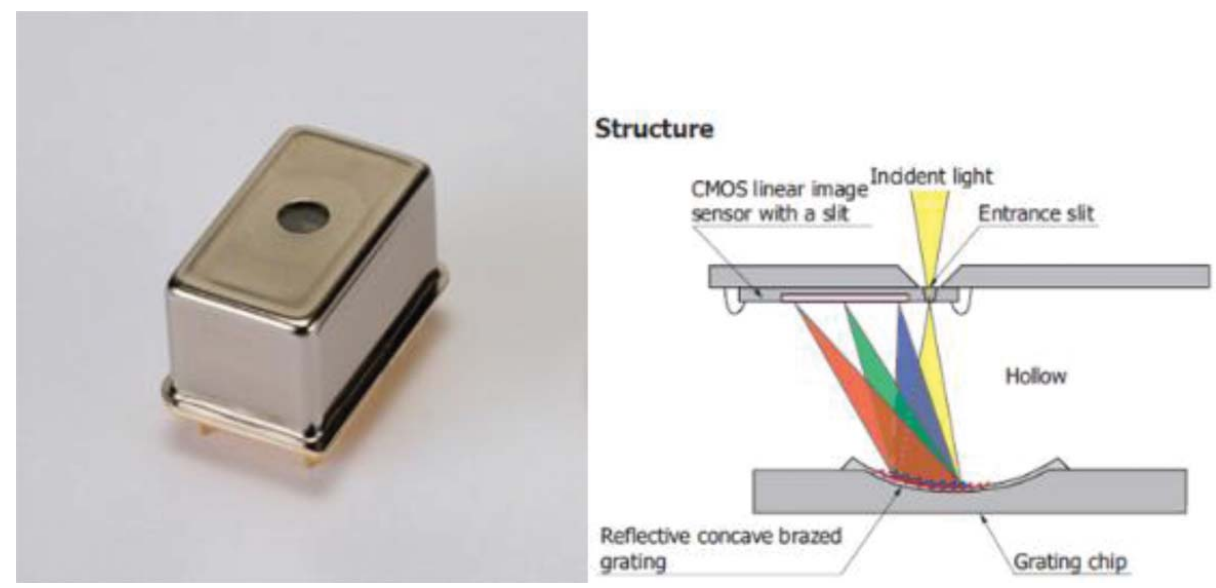

Figure 18. Basic scheme of finger-tip size spectrometer head (36). 


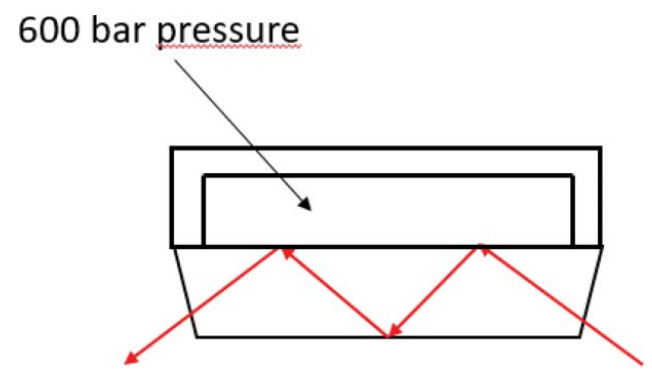

Figure 19. High-pressure microfluidics ATR-crystal system.

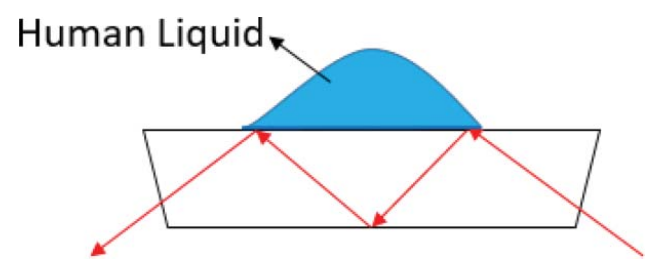

Figure 20. Biomedical applications with disposable Si ATR-IR.

Another application might be biomedical research and biomedical device fabrication. Disposable ATR crystals are useful for biomedical analysis (Figure 20), because disposable ATR crystals ensure superior hygienic conditions; a new crystal can be used for each sample. In addition, if disposable ATR crystals are employed, biomedical researchers may reuse samples, since IR spectroscopy is a non-destructive analysis. In this way, properties of the same sample can be measured by another analysis method. This is a significant improvement for biomedical research. Also, chemical reactions in confined spaces and confinement effects of molecules might be researched. Moreover, safety issues surrounding the study of explosive, highly toxic, and highly expansive reactions can be solved.

In forensic science, samples are gathered from crime scenes. The samples might be too limited for multiple analyses, so tests should be done carefully and samples should be preserved as much as possible. Disposable ATR-IR crystals allow the use of the same sample in more than one analysis since it is non-destructive. The samples can be preserved with disposable crystals so that a new analysis can be done or previous analysis can be repeated. Also, using handheld ATR-IR spectrometers, forensic scientists can analyze these samples at the crime scene (30).

\section{Conclusion}

Since the beginning of history, scientists have tried to explain what light is and how it can be used. In the 17th century, Newton discovered that white light was composed of a spectrum of different colors, and with prisms it was possible to divide it into its components. Although Newton himself described this phenomenon in different terms, this meant that white light contained a range of wavelengths. A few centuries later, quantum physics was developed, and scientists like Schrödinger, Feynman, de Broglie, and Einstein claimed that light had both particle and wave properties. This information precipitated improvements in spectrometric methods, such as UV-Vis, Raman and IR. IR spectroscopy is one of the most 
important and useful analytical methods in chemistry. Using IR radiation, IR active molecules can be detected easily, and samples can be reused since IR spectroscopy is a nondestructive method. Another advantage is that to perform the analysis, only a small portion of the sample is needed. However, it is not very accurate for aqueous solutions due to high absorptivity of water molecules in the IR region. Signals produced by water molecules hide or distort signals of other molecules. ATR-IR has solved this problem, so that we can take IR spectra accurately and precisely for aqueous solutions.

Currently, more ATR-IR spectroscopy techniques are being developed each day. For example, ATR crystals are being integrated with disposable Si-wafer crystals. These crystals can be changed easily without advanced technical tools. Therefore, they are cheaper than commercial disposable ATR crystals. Moreover, the crystals can be designed as microfluidic chips that allow analysis with even less sample. This may be especially advantageous to forensic scientists, who often must deal with limited samples. Disposable ATR crystals combined with miniature ATR-IR spectrometers allow easy transportation and analysis in many environments for forensic scientists and medical doctors, so that they can analyze body liquids immediately. Another way to improve this method may be to develop high-pressure miniature ATR-IR spectrometers for study of high-pressure reactions. It is difficult, expensive, and dangerous to install highpressure test equipment in large quantities. $\mu \mathrm{Si}$-ATR-IR would allow high-pressure material to be tested in very small volumes. This would be useful when studying explosive, highly toxic, and highly expansive reactions, or even yet untested reactions.

\section{Acknowledgement}

This work was supported by the Scientific and Technological Research Council of Turkey (TÜBİTAK), Grant No: 115E038.

\section{References}

1. Waldman, G. (2002) Introduction to light: The physics of light, vision, and color. Courier Corporation, Englewood Cliffs, NJ.

2. Young, H. D., and Freedman, R. A. (2015) University physics with modern physics. Addison-Wesley, Boston, Mass.

3. Corcoran, M. (2011) Quantum physics: A fundamental approach to modern physics. Am. J. Phys. 79(1): 143-144.

4. Pavia, D. L., Lampman, G. M., Kriz, G. S., and Vyvyan, J. A. (2008) Introduction to spectroscopy. Cengage Learning, USA.

5. Skoog, D. A. and West, D. M. (1980) Principles of instrumental analysis, vol. 158. Saunders College Publishing, Philadelphia.

6. Settle, F. (1997) Handbook of instrumental techniques for analytical chemistry. Prentice Hall PTR, Upper Saddle River, New Jersey.

7. Colthup, N. (2012) Introduction to infrared and Raman spectroscopy. Elsevier, USA.

8. Averill, B. A., and Eldredge, P. (2012) Principles of general chemistry. http://2012books.lard bucket.org/pdfs/principles-of-general-chemistry-v1.0.pdf.

9. Marengo, E., Liparota, M. C., Robotti, E., and Bobba, M. (2006) Monitoring of paintings under exposure to UV light by ATR-FT-IR spectroscopy and multivariate control charts. Vib. Spectrosc. 40(2): 225-234.

10. Friebe, A., and Siesler, H. W. (2007) In situ monitoring of an isocyanate reaction by fiber-optic FT-IR/ATR-spectroscopy. Vib. Spectrosc. 43(1): 217-220. 
11. Colthup, N. B., Daly, L. H., and Wiberley, S. E. (1990) Introduction to infrared and Raman spectroscopy. Academic Press, USA.

12. Blum, M. M., and John, H. (2012) Historical perspective and modern applications of attenuated total reflectance-fourier transform infrared spectroscopy (ATR-FTIR). Drug Test Anal. 4(3-4): 298-302.

13. Karabudak, E., Kas, R., Ogieglo, W., Rafieian, D., Schlautmann, S., Lammertink, R. G. H., Gardeniers, (Hen) J. G. E., and Mul, G. (2013) Disposable attenuated total reflection-infrared crystals from silicon wafer: A versatile approach to surface infrared spectroscopy. Anal. Chem. 85(1): 3338.

14. Karabudak, E., Mojet, B. L., Schlautmann, S., Mul, G., and Gardeniers, (Han) J. G. E. (2012) Attenuated total reflection-infrared nanofluidic chip with $71 \mathrm{~nL}$ detection volume for in situ spectroscopic analysis of chemical reaction intermediates. Anal. Chem. 84(7): 3132-3137.

15. Karabudak, E. (2014) Micromachined silicon attenuated total reflectance infrared spectroscopy: An emerging detection method in micro/nanofluidics. Electrophoresis 35(2-3): 236244.

16. Cocciardi, R. A., Ismail, A. A. and Sedman, J. (2005) Investigation of the potential utility of singlebounce attenuated total reflectance Fourier transform infrared spectroscopy in the analysis of distilled liquors and wines. J. Agric. Food Chem. 53(8): 2803-2809.

17. Vargas, A., Ferri, D., and Baiker, A. (2005) DFT and ATR-IR insight into the conformational flexibility of cinchonidine adsorbed on platinum: Proton exchange with metal. J. Catal. 236(1): 1-8.

18. Stepanyugin, A. V., Samijlenko, S. P., Martynenko, O. I., and Hovorun, D. M. (2005) ATR-IR spectroscopy as applied to nucleic acid films. Spectrochim. Acta A: Mol. Biomol. Spectrosc. 61(9): 2267-2269.

19. Pereira, A., Lopes, M., Timmer, J., and Keurentjes, J. (2005) Solvent sorption measurements in polymeric membranes with ATR-IR spectroscopy. J. Membr. Sci. 260(1-2): 174-180.

20. Marengo, E., Liparota, M. C., Robotti, E., Bobba, M., and Gennaro, M. C. (2005) Monitoring of pigmented surfaces in accelerated ageing process by ATR-FT-IR spectroscopy and multivariate control charts. Talanta 66(5): 1158-1167.

21. Luengo, C., Brigante, M., Antelo, J., and Avena, M. (2006) Kinetics of phosphate adsorption on goethite: Comparing batch adsorption and ATR-IR measurements. J. Colloid Interface Sci. 300(2): 511-518.

22. Fischer, D., Sahre, K., Abdelrhim, M., Voit, B., Sadhu, V. B., Pionteck, J., Komber, H., and Hutschenreuter, J. (2006) Process monitoring of polymers by in-line ATR-IR, NIR and Raman spectroscopy and ultrasonic measurements. C. R. Chim. 9(11-12): 1419-1424.

23. Bass, M., and Freger, V. (2015) Facile evaluation of coating thickness on membranes using ATRFTIR. J. Membr. Sci. 492: 348-354.

24. Ewing, A. V., Wray, P. S., Clarke, G. S., and Kazarian, S. G. (2015) Evaluating drug delivery with salt formation: Drug disproportionation studied in situ by ATR-FTIR imaging and Raman mapping. J. Pharm Biomed. Anal. 111: 248-256.

25. Popa, A. M., Angeloni, S., Burgi, T., Hubbell, J. A., Heinzelmann, H., and Pugin, R. (2010) Dynamic perspective on the function of thermoresponsive nanopores from in situ AFM and ATR-IR investigations. Langmuir 26(19): 15356-15365.

26. Kitadai, N., Yokoyama, T., and Nakashima, S. (2009) In situ ATR-IR investigation of L-lysine adsorption on montmorillonite. J. Colloid Interface Sci. 338(2): 395-401.

27. Kassis, A., Bhawtankar, V. M., and Sowa, J. R. Jr. (2010) Attenuated total reflection infrared spectroscopy (ATR-IR) as an in situ technique for dissolution studies. J. Pharm. Biomed. Anal. 53(3): 269-273.

28. Enders, D., Nagao, T., Pucci, A., and Nakayama, T. (2006) Reversible adsorption of Au nanoparticles on $\mathrm{SiO}_{2} / \mathrm{Si}$ : An in situ ATR-IR study. Surf. Sci. 600(6): L71-L75.

29. Dolamic, I., and Bürgi, T. (2011) In Situ ATR-IR Study on the photocatalytic decomposition of amino acids over $\mathrm{Au} / \mathrm{TiO}_{2}$ and $\mathrm{TiO}_{2}$. J. Phys. Chem. C 115(5): 2228-2234.

30. Orphanou, C. M. (2015) The detection and discrimination of human body fluids using ATR FTIR spectroscopy. Foren. Sci. Int. 252: e10-e16. 
31. Heise, H., and Bittner, A. (1995) Investigation of experimental errors in the quantitative analysis of glucose in human blood plasma by ATR-IR spectroscopy. J. Mol. Struct. 348: 21-24.

32. Whitesides, G. M. (2006) The origins and the future of microfluidics. Nature 442(7101): 368-373.

33. Herzig-Marx, R., Queeney, K. T., Jackman, R. J., Schmidt, M. A., and Jensen, K. F. (2004) Infrared spectroscopy for chemically specific sensing in silicon-based microreactors. Anal. Chem. 76(21): 6476-6483.

34. O’Brien, N. A., Hulse, C. A., Friedrich, D. M., van Milligen, F. J, von Gunten, M. K., Pfeifer, F., and Siesler, H. W. (2012) Miniature near-infrared (NIR) spectrometer engine for handheld applications. Paper presented at the Proceedings of SPIE.

35. Pyreos Sensing Solutions. (2017) Portable Pyreos ATR-IR. http://www.pyreos.com/line-arraycomponents.

36. Hamamatsu Photonics K.K. (2016) Micro-spectrometer. http://www.hamamatsu.com/jp/en/ C12666MA.html. 